\title{
IMPROVED TOPOGRAPHIC MAPPING THROUGH MULTI-BASELINE SAR INTERFEROMETRY WITH MAP ESTIMATION
}

\author{
Yuting Dong ${ }^{(1)}$, Houjun Jiang ${ }^{(1)}$, Lu Zhang ${ }^{(1),(2)}$, Mingsheng Liao ${ }^{(1),(2)}$, Xuguo Shi ${ }^{(1)}$ \\ (1) State Key Laboratory of Information Engineering in Surveying, Mapping and Remote Sensing, Wuhan University, \\ Wuhan, China, Email: yuting dong@whu.edu.cn \\ (2) Collaborative Innovation Center for Geospatial Technology, Wuhan, China, Email: luzhang@whu.edu.cn
}

\begin{abstract}
There is an inherent contradiction between the sensitivity of height measurement and the accuracy of phase unwrapping for SAR interferometry (InSAR) over rough terrain. This contradiction can be resolved by multi-baseline InSAR analysis, which exploits multiple phase observations with different normal baselines to improve phase unwrapping accuracy, or even avoid phase unwrapping. In this paper we propose a maximum a posteriori (MAP) estimation method assisted by SRTM DEM data for multi-baseline InSAR topographic mapping. Based on our method, a data processing flow is established and applied in processing multi-baseline ALOS/PALSAR dataset. The accuracy of resultant DEMs is evaluated by using a standard Chinese national DEM of scale 1:10,000 as reference. The results show that multi-baseline InSAR can improve DEM accuracy compared with single-baseline case. It is noteworthy that phase unwrapping is avoided and the quality of multi-baseline InSAR DEM can meet the DTED-2 standard.
\end{abstract}

\section{INTRODUCTION}

SAR interferometry (InSAR) is an effective tool for large-area topographic mapping due to its capability of all-time all-weather imaging and high sensitivity to terrain relief. However, for single-baseline InSAR, there is a contradiction between the sensitivity of height measurement and the accuracy of phase unwrapping over rough terrain. The height measurement sensitivity is proportional to normal baseline. However, large normal baseline usually implies high gradient of topographic phase and high geometric decorrelation, which decrease the accuracy and the reliability of phase unwrapping (Hanssen, 2001).

To solve this problem, multi-baseline InSAR has been proposed, which estimate terrain height by joint analysis of multiple phase observations with different normal baselines. It can reduce phase unwrapping error or even avoid phase unwrapping (Fornaro et al, 2005). In 1990 $\mathrm{Li}$ and Goldstein studied multi-baseline InSAR (Li et al, 1990), and since then researchers have proposed a variety of methods such as Chinese remainder theorem method (Xu et al, 1994), multi-baseline iterative estimation (Thompson et al, 1999), least squares estimation (Ghiglia et al, 1994), ML estimation
(Pascazio et al, 2001) and so on. The Chinese remainder theorem method is very sensitive to phase noise. The multi-baseline iterative method depends on accuracy of phase unwrapping and distribution of normal baseline. The least squares estimation method ignores the number and the location of phase discontinuities and hence the phase unwrapping error will propagate over the whole interferogram. The ML estimation method uses the probability distribution of interferometric phase to derive the terrain height. However, atmospheric effects (in repeat-pass case), orbital error and decorrelation will introduce significant error in ML estimation. The accuracy of ML estimation can be improved by introducing a priori information of terrain height, leading to the maximum a posteriori (MAP) estimation method.

In this paper, we propose a MAP estimation method assisted by SRTM DEM data to correct atmospheric effects and orbit error and to narrow the height search range. The priori information of terrain height is derived from SRTM DEM. In order to adapt the MAP method to spaceborne SAR data, we present a data processing flow including atmospheric effects correction for repeatpass interferogram. The method and the processing flow are applied on four ALOS/PALSAR interferograms.

\section{METHODOLOGY}

\subsection{MAP estimation assisted by SRTM DEM}

When the prior information of terrain height is available from SRTM DEM data, we can regard MAP estimation of multi-baseline InSAR as update of existing topographic information, namely to improve the resolution and accuracy of existing DEMs. The basis of our MAP estimation is: 1) $p d f(\phi \mid h)$, conditional probability density function of the interferometric phase $\phi$ for terrain height $h$ (i.e. the likelihood function of terrain height); 2) $p d f(h)$, priori distribution of terrain height. The estimation can be written as Eq. 1,

$$
\hat{H}_{M A P}=\arg \max _{h \in F}\left\{\left[\prod_{i=1}^{K} p d f\left(\varphi_{i} \mid h\right)\right] p d f(h)\right\}
$$

Under the premise of distributed scattering, the SAR return signal can be viewed as complex Gaussian random variables. Interferometric phase $\phi$ is the 
conjugate product of two complex Gaussian random signals and its marginal probability density function is Eq. 2 (Tough et al, 1995)

$$
\begin{aligned}
p d f(\phi \mid \phi, \rho, L)= & \frac{\left(1-|\rho|^{2}\right)^{L}}{2 \pi}\left\{\frac{(2 L-2) !}{[(L-1) !]^{2} 2^{2(L-1)}}\right. \\
& \times\left[\frac{(2 L-1) \beta}{\left(1-\beta^{2}\right)^{L+1 / 2}}\left(\frac{\pi}{2}+\arcsin \beta\right)+\frac{1}{\left(1-\beta^{2}\right)^{L}}\right] \\
& \left.+\frac{1}{2(L-1)} \sum_{r=0}^{L-2} \frac{\Gamma(L-1 / 2)}{\Gamma(L-1 / 2-r)} \frac{\Gamma(L-1-r)}{\Gamma(L-1)} \frac{1+(2 r+1) \beta^{2}}{\left(1-\beta^{2}\right)^{r+2}}\right\}
\end{aligned}
$$

Where $\phi_{0}$ is the expectation of the interferometric phase $\phi, L$ is the equivalent number of looks and $\rho$ is the complex correlation coefficient. $\beta=|\rho| \cos \left(\phi-\phi_{0}\right)$. Eq. 2 is the likelihood function of $\phi_{0}$. According to InSAR geometry, $\phi_{0}$ can be expressed by the target height $h$. As a result, the likelihood function of $\phi_{0}$ is converted to the likelihood function of $h$ (i.e. $p d f(\phi \mid h)$ ).

The priori distribution of terrain height $h$ can be derived from low-resolution reference DEM such as SRTM DEM, ASTER GDEM and so on. $h_{\text {DEM }}$ is the height of the reference DEM (Rodriguez et al, 2006). We assume that the systematic errors have been corrected and random errors obey Gaussian distribution with standard deviation $\sigma_{h}$ obtained from DEM accuracy assessment. It is worth mentioning that $\sigma_{h}$ is influenced by changes in local terrain. Thus the priori distribution of $h$ is defined as Eq. 3 .

$$
\begin{aligned}
p d f(h) & =\frac{1}{\sqrt{2 \pi \sigma_{h}^{2}}} \exp \left\{\frac{1}{T} \sum_{i \in \mathcal{N}} \frac{-\left(h-h_{\mathrm{DEM}, i}\right)^{2}}{2 \sigma_{h}^{2}}\right\} \\
\sigma_{h} & = \begin{cases}\sigma_{\text {local }}, & \sigma_{\text {local }}>\sigma_{\mathrm{DEM}} \\
\sigma_{\mathrm{DEM}}, & \sigma_{\text {local }} \leq \sigma_{\mathrm{DEM}}\end{cases}
\end{aligned}
$$

Where $\mathrm{N}$ is the neighbourhood system composed of the resolution cell and its neighbouring cells. For a given $\mathrm{N}$, T represents the number of cells, $h_{\mathrm{DEM}, \mathrm{i}}$ is the height of each cell and $\sigma_{\text {local }}$ is the standard deviation of heights.

\subsection{Processing flow of spaceborne SAR data}

To build processing flow for spaceborne SAR data, three issues are considered to enhance the reliability and the computing efficiency of the ML estimation, including: 1) the atmospheric effects and orbit error are corrected by using SRTM DEM; 2) rational polynomial model is developed for fast conversion between height $h$ and phase $\phi_{0}$. 3) the likelihood probability lookup table is used to replace complicated Eq. 2 for $p d f(\phi \mid h)$ calculation. Fig.1shows the processing flowchart:

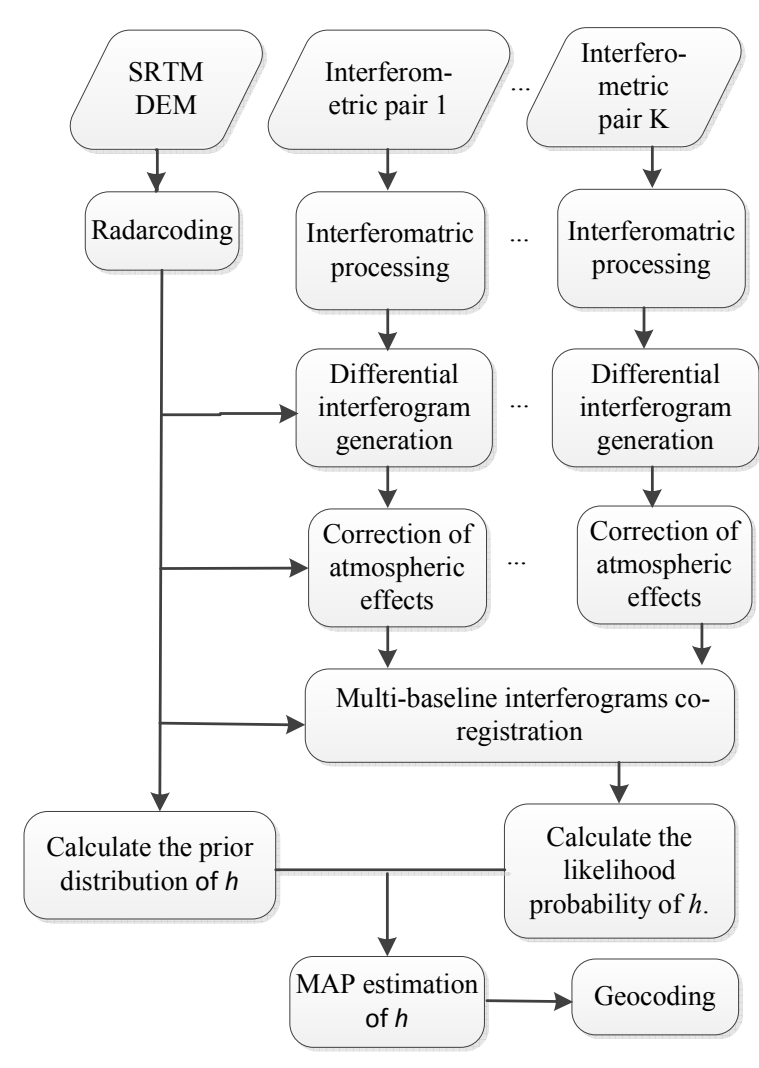

Figure 1. Multi-baseline InSAR processing flowchart for spaceborne SAR data

\section{EXPERIMENTAL RESULTS}

\subsection{Study area and datasets}

The proposed multi-baseline InSAR method is to be

\begin{tabular}{|c|c|}
\hline Image acquisition time & $\begin{array}{c}2007-12-22 / 2008-2-6 / 2008-3-23 \\
2009-12-27 / 2010-2-11 / 2010-3-29\end{array}$ \\
\hline Orbital direction & Ascending \\
\hline Imaging mode & Stripmap \\
\hline Polarization & $\mathrm{HH}$ \\
\hline Central incidence angle & $38.7^{\circ}$ \\
\hline $\begin{array}{l}\text { Azimuth/range spacing } \\
\text { space }\end{array}$ & $3.18 \mathrm{~m} / 4.68 \mathrm{~m}$ \\
\hline $\begin{array}{l}\text { Azimuth } / \text { range } \\
\text { bandwidth }\end{array}$ & $1522 \mathrm{~Hz} / 28 \mathrm{MHz}$ \\
\hline
\end{tabular}
tested on 6 L-band ALOS/PALSAR images over the areas of Tai'an city. The detailed parameters of these images are listed in Tab. 1.

Table. 1 Parameters of ALOS/PALSAR images 
The resolution of external SRTM DEM we used is about $90 \mathrm{~m}$ and the standard deviation of the global height error is about $5 \mathrm{~m}$ (Rodriguez et al, 2006). In order to verify the accuracy of multi-baseline InSAR DEM, we use 1:10,000 DEM data with spatial resolution of $5 \mathrm{~m} \times 5 \mathrm{~m}$ and height accuracy of $5 \mathrm{~m}$ provided by Shandong Province Institute of Land Surveying and Mapping. This DEM is generated by aerial photogrammetry and its coverage is about $12 \mathrm{~km}$ $\times 14 \mathrm{~km}$ including southern part of Mount Tai and Tai'an city area (shown as the green rectangle in Fig. 2).

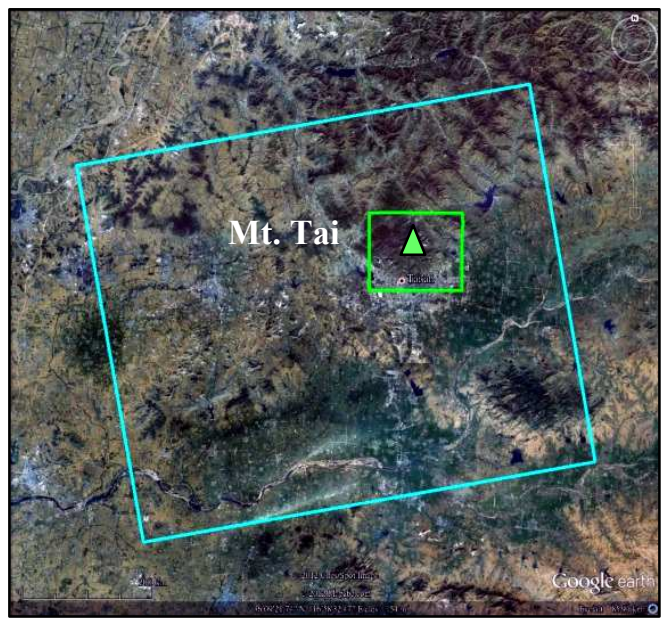

Figure 2. The coverage of experiment area(Google Earth). The cyan rectangle shows the coverage of $S A R$ image and the green shows the coverage of 1:10,000 DEM.

The experimental area is located at Tai'an City in the middle of Shandong Province and the coverage is about $58 \mathrm{~km} \times 70 \mathrm{~km}$, as shown in Fig. 2. The local topography is complicated, including plains, hills and mountains. The height changes substantially from 100 $\mathrm{m}$ (average height in plains) to $1533 \mathrm{~m}$ (height of Mountain Tai's peak), which is suitable for multibaseline InSAR experiments. Most part of this area is covered with vegetation. Hence we take advantage of ALOS/PALSAR's long wavelength signal which could keep good coherence and penetrate the vegetation to measure the surface height.

\subsection{Multi-baseline InSAR experimental results}

There are six ALOS/PALSAR images used in this experiment. Fig. 3 plots the temporal and spatial baseline distribution of the image data sets. We select four interferometric pairs connected by the blue lines. Image 2 and 5 are the master images for interferometric pair 1-2/2-3 and 4-5/5-6 respectively. Register 4-5/5-6 to the same image space with $1-2 / 2-3$ as shown in red line.

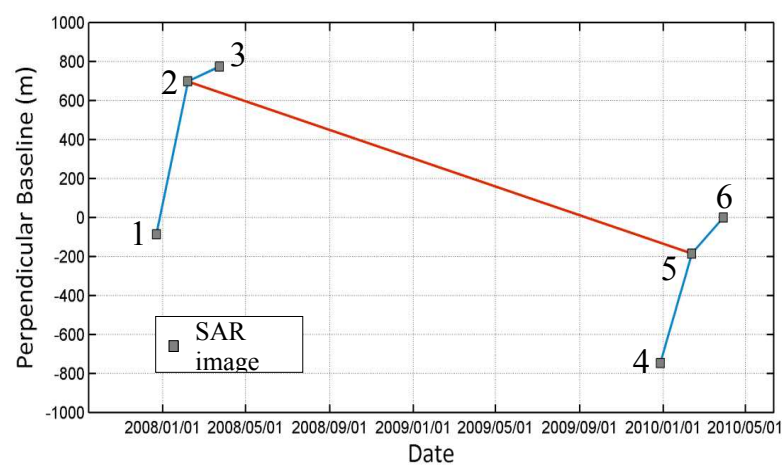

Figure 3. Temporal and spatial baseline distribution of ALOS/PALSAR image data sets

The parameters of the four interferograms are listed in Tab. 2 with the normal baseline ranging from $-784 \mathrm{~m}$ to $185 \mathrm{~m}$ and height ambiguity ranging from $82 \mathrm{~m}$ to 833 $\mathrm{m}$, forming a suitable combination of different baselines. Fig. 4 shows the interferograms of topographic phase. We could tell that with the increase of normal baseline, the fringe frequency becomes higher and the height ambiguity becomes lower especially in mountainous areas which makes phase unwrapping more difficult.

Table 2. Parameters of ALOS/PALSAR interferograms

\begin{tabular}{ccccc}
\hline & I & II & III & IV \\
\hline$B_{\text {temp }}$ (day) & 46 & 46 & 46 & 46 \\
$B_{\perp}(\mathrm{m})$ & -784 & 77 & -561 & 185 \\
$h_{2 \pi}(\mathrm{m})$ & 82 & 833 & 115 & 347 \\
$f_{D C}(\mathrm{~Hz})$ & $74 / 75$ & $74 / 80$ & $68 / 57$ & $68 / 46$ \\
$\rho$ & 0.52 & 0.53 & 0.58 & 0.50 \\
\hline
\end{tabular}

Where $B_{\text {temp }}$ is temporal baseline; $f_{D C}$ is the Doppler centroid frequency; $h_{2 \pi}$ is the height ambiguity; $\rho$ is average correlation coefficient.

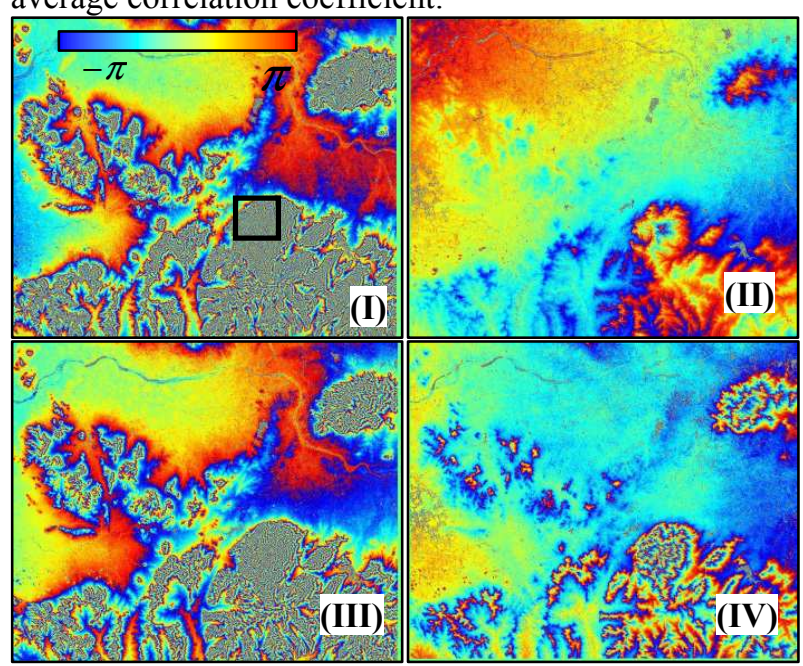

Figure 4. Interferograms of topographic phase. (I) 
$B_{\perp}=-784 m$, (II) $B_{\perp}=77 m$, (III) $B_{\perp}=-561 \mathrm{~m}$, (IV)

$B_{\perp}=185 \mathrm{~m}$. The black rectangle in (I) shows the coverage of elevation contrast area of Fig. 5.
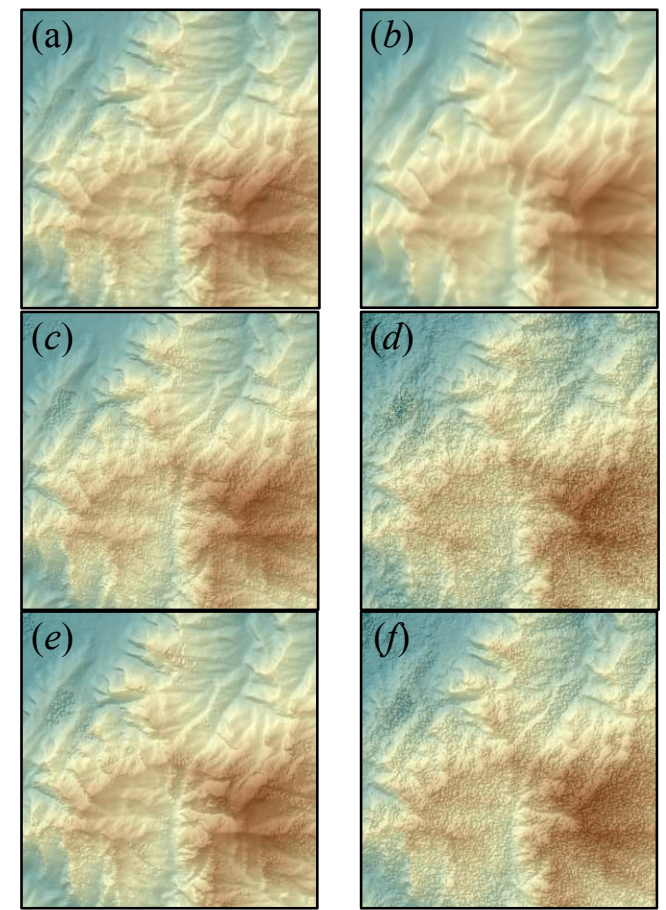

Figure 5. Height maps of Mount Tai in radar coordinates. (a) DEM generated by MAP estimation, (b) radarcoded SRTM DEM, (c)-(f) DEM generated by interferogram I-IV respectively.

From comparative analysis of these six height maps in Fig.5, we could see the DEM generated by MAP estimation (Fig. 5 (a)) has lower noise level than DEM generated by single interferogram (Fig. 5 (c) - (f)). Fig. 5(d) and (f) are very noisy due to high height ambiguity of interferogram II, IV. The mountainous areas of Fig. 5(c) are nosier than Fig. 5(a) due to geometric decorrelation caused by long normal baseline of interferogram I. Fig. 5 (a), (c), (e) have better resolution than radarcoded SRTM DEM(Fig. 5(b)).

\subsection{InSAR DEM quality evaluation}

The DEMs generated by multi or single baseline InSAR are geocoded with WGS 84 as the geodetic datum and EGM 96 as the gravity reference and then projected into the UTM coordinate system with spatial resolution of 20 m. Fig. 6 shows the hill-shaded multi-baseline InSAR DEM with height ranging from $20 \mathrm{~m}$ to $1535 \mathrm{~m}$. We can see the mountains and hills clearly.

We reshape the resolution cell size of 1:10,000 DEM from $5 \mathrm{~m}$ to $20 \mathrm{~m}$. Then we calculate the differences between multi/single baseline DEMs, SRTM DEM and reshaped 1:10,000 DEM respectively.

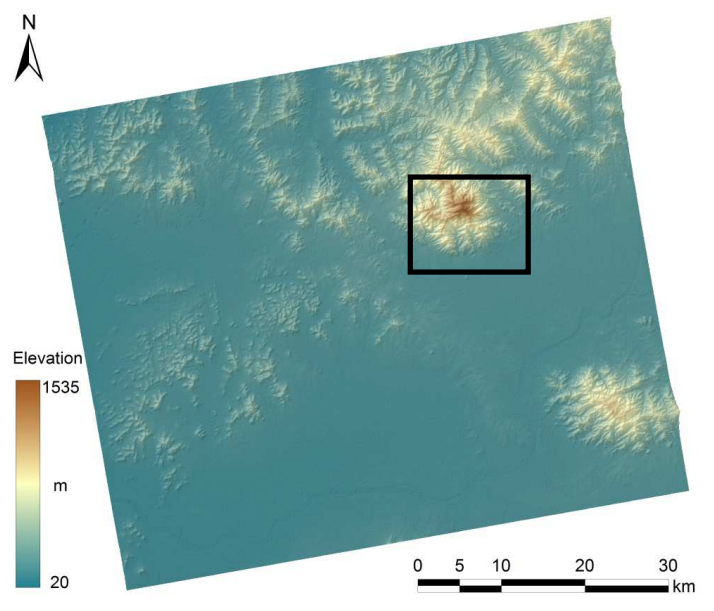

Figure 6. Hill-shaded multi-baseline InSAR DEM. The black rectangle shows the coverage of 1:10,000 DEM.
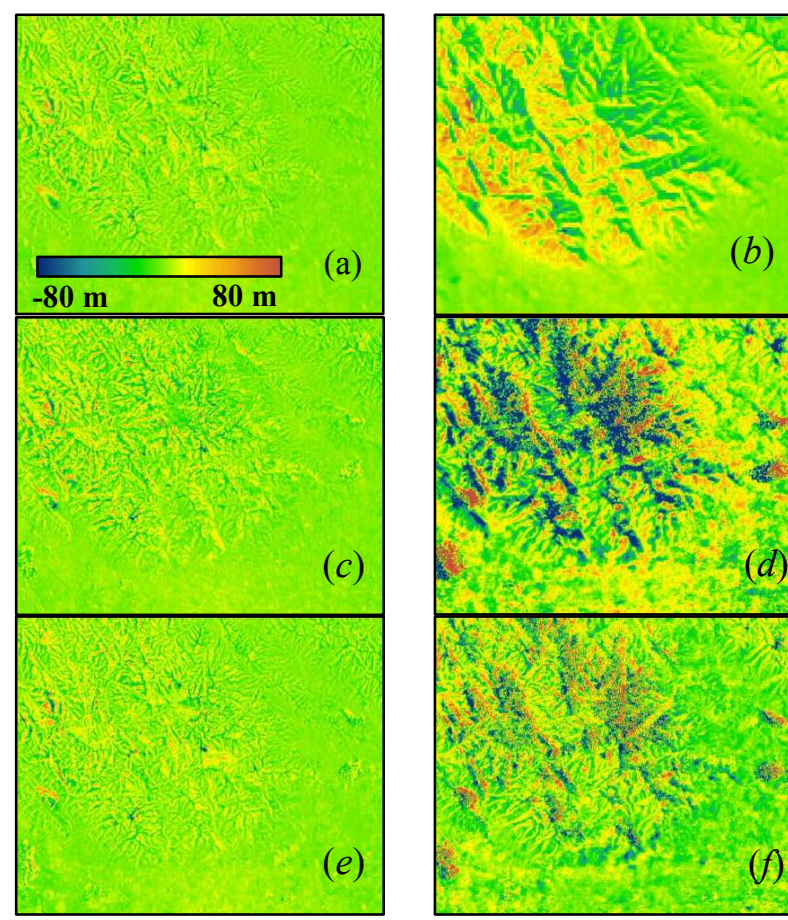

Figure 7. Height difference maps between InSAR DEM, SRTM DEM and 1:10,000 DEM, (a) DEM generated by MAP estimation, (b) radarcoded SRTM DEM, (c)-(f) DEM generated by interferogram I-IV respectively.

We can see from Fig. 7 that the height error of multibaseline InSAR DEM (Fig. 7(a)) is much smaller than that of single-baseline InSAR DEMs or SRTM DEM (Fig. 7 (c)-(f), (b)), especially in mountainous areas. With the decrease of the normal baseline, the height error becomes larger for single baseline DEM. The spatial distribution of height error of multi-baseline InSAR DEM is similar with DEMs generated by interferogram I, III which have long normal baselines and high height accuracy. As for SRTM DEM (Fig.7 (b)), the higher the height changes, the larger the height error could be. This is caused by the low resolution of 
SRTM DEM and it cannot depict enough details of terrain. Compared with SRTM DEM, multi-baseline InSAR DEM has more advantages in terms of resolution. Finally, we quantitatively evaluate the height accuracy. Tab. 3 shows the statistics of the differences between multi/single baseline InSAR DEMs, SRTM DEM and 1:10,000 DEM. For multi-baseline DEM, the standard deviation is the smallest and the portion of absolute height errors less than $10 \mathrm{~m}$ is the largest compared with other DEMs. The height accuracy of single baseline DEM is determined by their height ambiguity. The multi-baseline DEM could be viewed as update of the SRTM DEM for its improvement in both height accuracy and resolution. Assuming that the height errors obey Gaussian distribution, the absolute height error of multi-baseline InSAR (90\% linear error) is about $14.1 \mathrm{~m}$ which is less than that of the DTED-2 standard (18 m). The spatial resolution of DTED-2 standard is $30 \mathrm{~m}$ $\times 30 \mathrm{~m}$ which is coarser than that of multi-baseline InSAR DEM $(20 \mathrm{~m} \times 20 \mathrm{~m})$. Hence we could say the multi-baseline InSAR DEM meets the DTED-2 standard from perspective of spatial resolution and height accuracy.

Table 3. Height difference statistics

\begin{tabular}{cccc}
\hline & $\Delta h_{\text {ave }} / \mathrm{m}$ & $\sigma_{\Delta h} / \mathrm{m}$ & $|\Delta h| \leq 10 \mathrm{~m}$ \\
\hline$\Delta h_{I}$ & 1.9 & 11.3 & $81.4 \%$ \\
$\Delta h_{I I}$ & -4.4 & 43.0 & $32.8 \%$ \\
$\Delta h_{I I I}$ & 2.3 & 10.6 & $83.0 \%$ \\
$\Delta h_{I V}$ & -0.3 & 27.7 & $51.8 \%$ \\
$\Delta h_{M A P}$ & 1.7 & 8.6 & $86.3 \%$ \\
$\Delta h_{S R T M}$ & 4.9 & 15.4 & $58.9 \%$ \\
\hline
\end{tabular}

Where $\Delta h_{I-I V}, \Delta h_{M A P}$ and $\Delta h_{S R T M}$ are height differences between DEMs generated by interferogram I-IV, multibaseline DEM, SRTM DEM and 1:10,000 DEM respectively; $\Delta h_{\text {ave }}$ and $\sigma_{\Delta h}$ are the average and the standard deviation of height differences.

\section{CONCLUSIONS}

In this paper we propose a MAP estimation method assisted by SRTM DEM data for multi-baseline InSAR topographic mapping to generate high-quality DEM and avoid phase-unwrapping. External low-resolution DEM could provide a priori information of height distribution and effectively improve the reliability of DEM generation. The atmospheric effects cannot be ignored in repeat-track InSAR and should be removed from single interferometric pairs. The DEM generated by ALOS/PALSAR high resolution interferometric data could meet DTED-2 standard.

\section{ACKNOWLEDGEMENTS}

This work was financially supported by the National Key Basic Research Program of China under Grant 2013CB733204, the National Natural Science Foundation of China under Grants 61331016 and 41271457, and the Shanghai Academy of Spaceflight Technology Innovation Fund under Grant SAST201321. The authors thank the Japan Aerospace Exploration Agency (JAXA) for providing the PALSAR data through ALOS RA-4 projects (PI 1247 and 1440).

\section{REFERENCES}

1. Hanssen, R.F., 2001. Radar Interferometry: Data Interpretation and Error Analysis. KluwerAcademic Publisher, Dordrecht, The Netherlands.

2. Fornaro, G., Monti Guarnieri, A., Pauciullo, A., Tebaldini, S., 2005.JointMulti-baseline SAR Interferometry, EURASIP Journal on Applied Signal Processing, 2005, 3194-3205.

3. Li, F. K., Goldstein, R. M., 1990, Studies of MultibaselineSpaceborne Interferometric Synthetic Aperture Radars, IEEE Transactionson Geoscience and Remote Sensing, 28(1), 88-97.

4. Xu, W., Chang, E. C., Kwoh, L. K., Lim, H., Cheng, W., Heng, A., 1994.Phase Unwrapping of SAR Interferogram with Multi-frequency or Multibaseline, Geoscience and Remote Sensing Symposium 1994 (IGARSS'94), Vol.2, 730-732.

5. Thompson, D. G., Robertson, A. E., Arnold, D. V., Long, D. G., 1999.Multi-baseline interferometric SAR for Iterative Height Estimation, Geoscience and Remote Sensing Symposium 1999 (IGARSS'99), Vol.1, 251-253.

6. Ghiglia, D. C., Wahl, D. E., 1994. Interferometric Synthetic Aperture Radar Terrain Height Mapping from Multiple Observations, IEEE Digital Signal Processing Workshop 1994, 33-36.

7. Pascazio, V., Schirinzi, G., 2001. Estimation of Terrain Height by Multifrequency Interferometric Wide Band SAR Data, IEEE Signal Processing Letters, 8(1), 7-9.

8. Tough, R. J. A., Blacknell, D.,Quegan, S., 1995. A Statistical Description of Polarimetricand Interferometric Synthetic Aperture Radar, Proceeedings of the Royal SocietyLondon A, 449, 567-589.

9. Rodriguez, E., Morris, C. S., Belz, J. E., 2006, A Global Assessment of the SRTMPerformance, Photogrammetric Engineering \& Remote Sensing, 72(3), 249-260. 